\title{
05.
}

\section{Melancholia in the Time of War: Analysing the Efficacy of Psychosocial Aid in Post-Conflict Liberia}

Trisha Bharti, SYBA

\section{Introd uction}

The sub-Saharan country of Liberia in West Africa is notorious for its profusion of wars and conflicts in history that supervened the nation, and left them with the onus of a post war reconstruction. The first leg of the Liberian Civil War broke out in 1989 , when the country welcomed the election of a native Liberian leader, Samuel Doe, whose regime ultimately incorporated authoritarian, repressive and discriminatory tactics. In consequence, his government was overthrown by the group under politician and guerilla leader Charles Taylor, called the Independent National Patriotic Front of Liberia (INPFL). Succeeding the rebellion, Taylor was eventually elected as president. The period of quietude experienced by the citizens following the first war proved to be rather ephemeral, as the latter war burgeoned in 1999, a mere 2 years after the former ended. The second Liberian Civil War transpired when Taylor's forces were pitted against two armed rebel groups hailing from northern Liberia : Liberians United for Reconciliation and Democracy (LURD) and the Movement for Democracy in Liberia (MODEL).

\section{Impact of the Wars}

The cataclysmic wars ensued the deaths of over 250,000 civilians; and the displacement of about a million led to an extreme debilitation in political, economic, humanitarian, and social conditions. The conflicts were infamous for having recruited over 15,000 children who fought for the government, in addition to young girls being abducted and forced into sexual servitude. The distressed ex-combatants and affected civilians, bereft of basic necessities, inevitably suffered from a myriad of mental health disorders like major depressive disorders, Post-traumatic stress disorder (PTSD), suicidal tendencies etc. They consequently indulged in extreme antisocial behaviours such as theft, torture, rape, killing, physical and substance abuse, etc.

Additionally, subpar standards of living, loss of lives, poverty and unemployment encountered by civilians further debilitated their mental state, and impairing dimensions of social, cognitive, educational, economic and functional development. In 2010, Liberia stood 162 out of 169 countries in the Human Development Index (HDI), deeming it to be one of the poorest nations in the world.

\section{Psychosocial Aid in Post-Conflict Liberia}

The egregious ramifications of the conflicts called for a complete restructure of the nation, with the reparations of collapsed healthcare systems being of primary importance. The Liberian post conflict society, progressively, has observed significant improvements throughout different spheres, with an aim to set the stage for a promising future.

As a response to the post-war health problems, community-based mental health programs garnered significant importance and utilised two approaches to engage with local communities; The first one requiring Mental Health and Psychosocial Services (MHPSS) to be integrated into various sectors by placing mental health workers in communitybased trauma healing and counselling. In the second approach, the Ministry of Health and Social Welfare, with aid from donors and various NGOs, moved forward with the provision of a basic package of healthcare services, especially in rural areas, that 
included mental health counselling and treatment.

The latter half of the post-war decade saw intimate psychiatric care as a priority, facilitating the establishment of many psychiatric residency training programs in partnership with the University of Liberia and Massachusetts General Hospital. Liberia, in 2010, also became a member of the Mental Health Leadership and Advocacy Program (mhLAP), an initiative promoting mental health policies and its practice.

Additionally, UNICEF prioritized inclusion of psychosocial services in the education sector in order to deem the institutions important for post-conflict reconstruction and ex-combatant rehabilitation. Many higher educational institutes, such as the University of Liberia, welcomed large classes of ex-combatants over the last decade, who were provided with psychosocial support to partake in the broader rehabilitation process. The postwar period has also recognised the development of formal 'grassroots organizations' and social support groups for people with mental disparities, appealing to the communalism and collectivistic culture in the citizens.

\section{Analysing the Efficacy of Psychosocial Aid}

Despite the monumental feats the various sectors achieved in the post conflict reconstruction, the mental health system in Liberia being deeply under-resourced and timid is a fact that yet stands. Immediately after the calamitous conflicts, Liberia witnessed the absconder of doctors, nurses and health-care professionals. This left a debilitating nation of 3 million to bear the brunt of the trauma caused from the wars, both physical and mental, by themselves.

In the Disarmament, Demobilization, Rehabilitation, and Reintegration (DDRR) process that commenced immediately after the second civil war, all activities under it required inclusion of psychiatric components. However, the nascent program encountered an inability to establish a Liberian psychiatrist. In addition to this, poor funding, lack of preparation of staff and trained professionals and management resulted in the absence of any effective mental health or psychosocial screening. This inevitably left the ideation and implementation of such commendable services redundant.

A vestige of ignorance towards the psychosocial processes and mental health illnesses and a lack of regard toward the consequences of the same is portrayed by the government and privatesectors. This can be witnessed in the financing of mental health care in the Liberian primary healthcare program, which has taken a low priority as it is not considered important to treat as other physical maladies and conditions. Additionally, the availability of specialised diagnostic and counselling skills, multiple follow ups and deployment of such interventions are significantly beyond Liberia's constricted tenuous resources due to it being a nascent program. As if putting together these components and getting them into gear is not an easy task (especially for a nation with incipient health care emerging from years of conflict), the lack of, and the inefficacy in execution and management of the programmes adds to restrictions in these services.

Often, psychosocial programs train counsellors and therapists inefficiently, leading to informal approach, unskilled practises and overall inconsistencies across the counselling sector. This may be further impeded by the ostensible risks of vicarious traumatization and lack of adequate resources for the psychosocial workers, who are often neophytes in the field and are especially young. In addition, they themselves are mentally unfit and have their own trauma to overcome, thus, making it difficult for them to help others. Lekskes and colleagues, for instance, have stated, "Counselors may not be able to provide professionally warranted counseling and are restricted to the level of 'good neighbors' advice" (2007, pg.24).

Liberia's efforts to reinvigorate the healthcare system is additionally hampered by a low budget that is highly dependent on the financing of international donors (approximately $80 \%$ of the nation's expenses on the healthcare system was financed by international 
donors in 2008). Such a collaboration with a huge number of donors can lead to colossal challenges in coordination, management, and execution by the government. Further, the ignorance of the NGOs when it comes to the topic of mental health is conspicuous as it is witnessed that most primary healthcare facilities run by international NGOs do not include mental health in their programmes.

Mental health illnesses and disorders are subjected to severe stigmatisation among the Liberian society, which believes that mental illness is contagious, relegating the sufferers to corporal punishment, social ostracization, substance and alcohol abuse to self-medicate (evident in a 2008 study by Johnson et al, which found ex-combatant's propensity to abuse alcohol, suggesting a probable attempt at selfmedicating mental illness). Such coping mechanisms can do more harm than good, and may amplify the sufferer's mental disparities further.

In a society that pays high regard to familial and communal relations, a lack of community support can hinder the accessibility of individuals with mental disorders to healthcare. It is widely known that societal pressures tend to inflict major psychological distress, and this may aggravate the individual's condition further. Community based approaches toward psychosocial aid, which possess the upper hand of drawing upon the collectivistic culture endemic in Liberia, fail to properly reinforce their programs and reduce stigma and discrimination for the same due to inept workers and ineffective management.

Another factor to consider is the inability to avail the extant mental health care facilities. Poor roads, infrastructure and travelling difficulties in Liberia, especially in the rural areas, make even nearby facilities difficult to reach. A testament of this is seen in the Demographic and Health Survey report that remarked that around $70 \%$ of women adjudged the distance between them and the medical facilities as a major hindrance to accessing health care. To further reinforce this, a study conducted by Kruk and colleagues which aimed to "assess the availability of essential health services in northern Liberia" in 2008 , calculated that only $12.1 \%$ could avail mental health services at their nearest facility (2008, p.5). This predicament further highlights the ravaged conditions of rural areas.

Through the plethora of aforementioned examples, it is evident that the Liberian government's programmes towards the treatment of mental health illnesses only look good on paper, and the inadequacy towards the ramifications and planning of these policies is heavily apparent. The same can be observed of the private sectors as well, who solely stress on the importance of physical health care, and simultaneously disregard the gravity of mental health.

\section{Eval uating Liberia's Present Condition}

After almost two decades of gruelling conflict and destruction, Liberia's post-war reconstruction might not be the most effectively calculated initiative, but it is on its way to achieve a nation free from the shackles of poverty, insecurities, conflicts and terrors.

Presently, the Liberian citizens have resorted to publicly demand for more accessibility to mental health support. This is due to the continuous realisation that mental health care is sidelined and that private aid is inefficient to assure assistance for every Liberian. From this, one can easily interpret that although the Liberian government has introduced a miscellany of policies and interventions to alleviate mental health illness across the country, it has yet not seen fit to make the same an increasing priority.

Nevertheless, The Ministry of Health's Mental Health Policy and Strategic Plan for 2016-2021 outlines key areas for improvement and aims at consolidating the nation's mental health legislation, expanding psychosocial worker training, and encouraging stigma reduction. Some of these policies include increasing the intake of mental health professionals and in-patients by the consolidation of wellness unit's throughout the county hospitals, expanding availability of mental health clinicians, promoting anti-stigma and anti-discrimination efforts and many more.

\section{Conclusion}

Although the novel policies display notable 


\section{Research Articles}

ambition and advocate nothing short of a significant restructure in the healthcare system, it is realistic to assume that the Liberians won't be dwelling in the comfort of a Xanadu anytime soon. The government's bold and resilient efforts need to be surveilled with the utmost care to ensure sustenance in the mental health services and fabrication of an efficient health system that can placate mental health and psychosocial burdens to the best of its abilities for a more secure and promising tomorrow.

The Liberian nation has endured long-standing conflicts that have inevitably put a dent on the calm and peace in the quotidian lifestyle of the citizenry, leaving them to inadvertently falter under the weight of such acrimonious consequences. For a government that promises a state of order, it is of utmost importance and desideratum to reinstate the idea of peace back into society. However, as long as conflict within oneself cannot be mended and as long as the community is still under tremendous mental distress, one could contend that establishing a state of calm and reviving the nation to its halcyon days will most definitely be a herculean task.

\section{References}

Abramowitz, S. (n.d.). WHO-Liberia Mental Health Primer.pdf. Academia.Edu. Retrieved January 14, 2021, from https://www.academia. edu/33262435/WHO_Liberia_Mental_Health_ Primer_pdf?email_work_card=view-paper

Abramowitz, S. A. (2014). Searching for Normal in the Wake of the Liberian War (Pennsylvania Studies in Human Rights) [E-book]. University of Pennsylvania Press. https://www.researchgate.net/ publication/274708332_Searching_for_Normal in the Wake_of the Liberian_War

Atkinson, P. A. (1997, May). The War Economy in Liberia : A Political Analysis. RELIEF AND REHABILITATION NETWORK. https:// odihpn.org/wp-content/uploads/1997/05/ networkpaper022.pdf

Esan, O. (n.d.). Global Mental Health Reforms: Mental Health Care in Anglophone West Africa. Academia.Edu. https://www.academia.
edu/22099437/Global_Mental_Health_Reforms Mental_Health_Care_in_Anglophone_West Africa?email_work_card=reading-history

Galea, Rockers, S. G. P. R. (n.d.). Persistent Psychopathology in the Wake of Civil War: LongTerm Posttraumatic Stress Disorder in Nimba County, Liberia. NCBI. https://www.ncbi.nlm.nih. gov/pmc/articles/PMC2920997/

Kruk, M. E. (2010, July 1). SciELO - Saúde Pública Availability of essential health services in post-conflict Liberia Availability of essential bealth services in post-conflict Liberia. SciELO. https://www.scielosp. org/article/bwho/2010.v88n7/527-534/en 